\title{
STEM-Technology Example of the Computational Problem of a Chain on a Cylinder
}

\author{
Valery Ochkov ${ }^{1}$, Konstantin Orlov ${ }^{2}$ \\ National Research University \\ Moscow Power Engineering Institute \\ Moscow, Russia \\ Evgeny Barochkin ${ }^{3}$ \\ Ivanovo State Power Engineering University \\ Ivanovo, Russia
}

\author{
Inna Vasileva ${ }^{4}$ \\ N.E. Zhukovsky and Y.A. Gagarin Air Force Academy \\ Voronezh, Russia
}

\author{
Evgeny Nikulchev ${ }^{5}$ \\ MIREA — Russian Technological University \\ Moscow, Russia
}

\begin{abstract}
An application of the STEM technology to the computational problem of the parameters of a closed chain (with and without load) thrown over a horizontal cylinder is considered. The numerical solution is found and its graphical interpretation is made by compiling a system of transcendental equations, as well as carrying out numerical optimization with constraints. The approximating analytical dependence is determined using the fitting functions. In the process of solving a number of concepts from mathematics, physics, computer science are examined. Some possibilities of using specialized mathematical packages (in particular, Mathcad) and of working on online platforms are shown. Additional problems options for using STEM technology are presented.
\end{abstract}

Keywords-STEM technology; math education; closed chain; Mathcad.

\section{INTRODUCTION}

Modernization of higher education is based on the use of new technologies [1]. For engineering and mathematical educations an important area is STEM technology training [26]. The problem of the parameters of a closed chain (with and without load) thrown over a horizontal cylinder, showing the application of the STEM technology in education [7-10], is considered.

At first the case of a closed chain without a pendant is analysed. The numerical solution and its graphical interpretation are given using the Mathcad Prime package. By working on online platforms an approximating function is constructed.

Then the more complex case of a closed chain with a pendant is modelled.

In terms of this article a well-known problem of a chain is examined using physics, mathematics, resistance of materials, hydro-gas dynamics, heat transfer, etc.

In solving this problem, a number of concepts from the following disciplines are used [11, 12]:

- Mathematics: function, derivative, integral, antiderivative, curve length, center of gravity of the

\section{A. Formulation of the Problem}

A closed chain of length $L$ is thrown onto a horizontal cylinder of radius $\mathrm{R}$. Determine the shape of the sagging chain, whose length, naturally, is greater than the circumference of the cylinder: $\mathrm{L}>2 \pi \mathrm{R}$.

This problem is attractive because it can be formulated not only in words, but also tested physically. To do this, simply attach a cylindrical disk to the board in a classroom, put a closed chain on it (see Fig. 1), take a picture of the sag of the chain with a digital camera, process the image on a computer, and then compare with the corresponding mathematical model (i.e. compare the real object with its digital double). This would make an excellent student laboratory activity.

Fig. 2 shows a scheme of the problem. Choose the origin at a point directly below the center of the circle at a distance $h$ from it. This unknown quantity, along with the other two (xo and a) described below, is the object of the search. Another important search parameter is the angle $\theta$ - the angle at which the chain breaks away from the cylinder.

\section{B. Numerical Solution}

Nowadays, such problems are increasingly being solved numerically, with the use of mathematical computer programs, rather than analytically $[13,14]$. In this article the Mathcad Prime computational package is used [15]. The requirement is to find a formula through which the angle $\theta$ is calculated depending on the ratio of $\mathrm{L}$ to $\mathrm{R}$. Fig. 3 shows how the six 
functions required to solve the problem are specified in Mathcad Prime.

The canonical catenary formula is $\mathrm{a} \cdot \cosh (\mathrm{x} / \mathrm{a})[16,17]$. It has a minimum (sagging chain: a $>0$ ) or maximum (arch: a < 0 ) at the point with abscissa equal to zero and the ordinate equal to a (see Fig. 2). The value of this parameter a needs to be found. The quantity a is also a certain "steepness" of the chain line: if a tends to zero, then the curvature of the chain also tends to zero, it turns into a stretched string. Another unknown is the variable $\mathrm{x} 0$ : the abscissa of the separation point of the chain from the cylinder (see Fig. 1). There are two such points, but they are symmetric with respect to the ordinate axis.

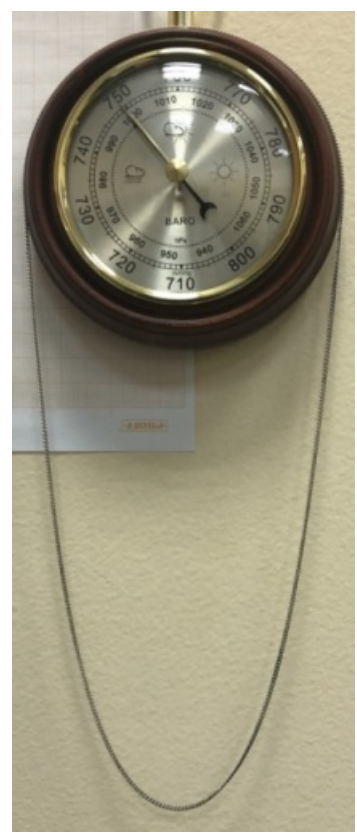

Fig. 1. A Chain Wrapped around a Wall-Mounted Aneroid Barometer.

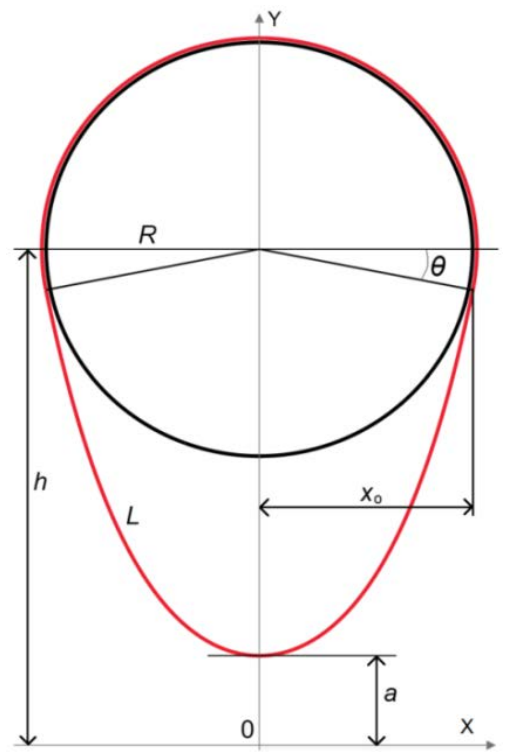

Fig. 2. Scheme of the Problem of the Chain on the Cylinder.

$$
\begin{aligned}
& F_{c}(x, a):=a \cdot \cosh \left(\frac{x}{a}\right) \text { Catenary } \\
& F_{c}^{\prime}(x, a):=\frac{\mathrm{d}}{\mathrm{d} x} F_{c}(x, a) \rightarrow \sinh \left(\frac{x}{a}\right) \quad \begin{array}{l}
\text { Derivative } \\
\text { of a catenary }
\end{array} \\
& S_{c}\left(x_{0}, a\right):=\int_{-x_{0}}^{x_{0}} \sqrt{1+F_{c}^{\prime}(x, a)^{2}} \mathrm{~d} x \quad \begin{array}{l}
\text { Catenary length } \\
\text { from - } x_{\mathrm{o}} \text { to } x_{\mathrm{o}}
\end{array} \\
& F_{\circ}(x, R, h):=h-\sqrt{R^{2}-x^{2}} \quad \text { Lower semicircle formula } \\
& F_{\circ}^{\prime}(x, R):=\frac{\mathrm{d}}{\mathrm{d} x} F_{0}(x, R, h) \rightarrow \frac{x}{\sqrt{R^{2}-x^{2}}} \begin{array}{l}
\text { Derivative lower } \\
\text { semicircles }
\end{array} \\
& S_{\circ}\left(x_{0}, R\right):=R \cdot\left(\pi+2 \text { acos }\left(\frac{x_{0}}{R}\right)\right) \quad \begin{array}{c}
\text { Chain length lying } \\
\text { on the cylinder }
\end{array}
\end{aligned}
$$

Fig. 3. Auxiliary Functions of the Problem of the Chain on the Cylinder.

The definite integral, which sets the length of the catenary, could be simplified through finding the antiderivative. This work is now being done more and more often using the Internet, e.g. www.wolframalpha.com (see Fig. 4).

As can be seen from Fig. 4, it is not possible to take a specific integral using Wolframalpha (the original expression is returned). But if you work with an indefinite integral, then the problem will be solved (last line in Fig. 4.) After that, it suffices to use the Newton-Leibniz theorem and get the desired chain length formula from -xo to xo: 2a· $\sinh (x o / a)$ (see Fig. 5, where the csgn function is the sign of the argument, in this case it is positive).

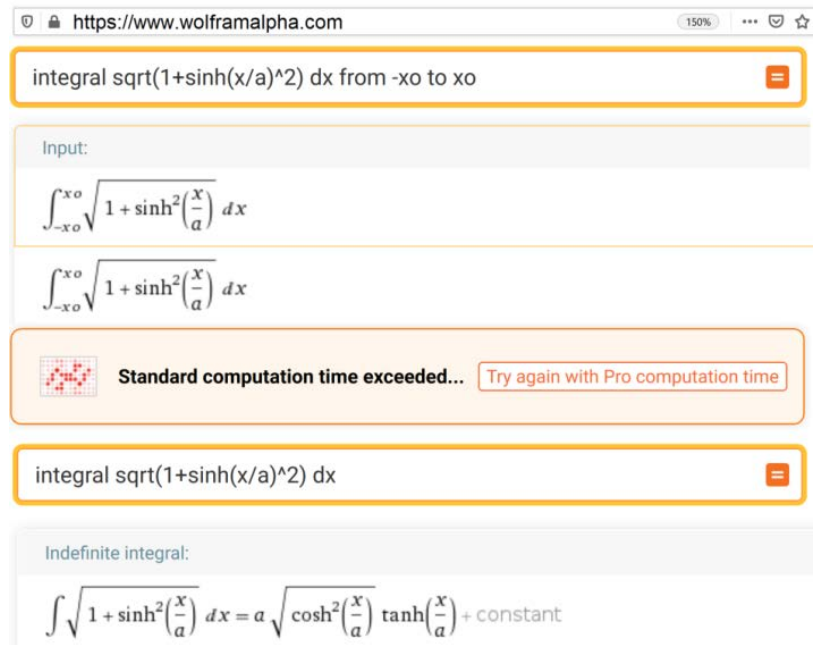

Fig. 4. Internet Search for the Primitive Function.

$$
\begin{aligned}
& \operatorname{int}(x, a):=a \cdot \sqrt{\cosh \left(\frac{x}{a}\right)^{2}} \cdot \tanh \left(\frac{x}{a}\right) \\
& \operatorname{int}\left(x_{o}, a\right)-\operatorname{int}\left(-x_{o}, a\right) \stackrel{\operatorname{simplify}}{\longrightarrow} \\
& \longrightarrow a \cdot \sinh \left(\frac{x_{o}}{a}\right) \cdot \operatorname{csgn}\left(\cosh \left(\frac{x_{o}}{a}\right)\right)+a \cdot \sinh \left(\frac{x_{o}}{a}\right) \cdot \operatorname{csgn}\left(\cosh \left(\frac{x_{o}}{a}\right)\right)
\end{aligned}
$$

Fig. 5. Simplification of the Catenary Length Formula. 
Fig. 6 shows the continuation of Mathcad calculation: the input data and the calculation of an important parameter of the problem - the ratio of the chain length $L$ to the circumference of the cylinder $2 \pi R$, on which the chain is thrown. This parameter can vary from one (the chain fits the cylinder tightly: $\theta=90^{\circ}$-see Fig. 2) to infinity (the lashes of the chain sag almost vertically at the circle: $\theta=0$ ).

The solution of the problem is reduced to the numerical search for the root of a system of three transcendental equations. This operation in Mathcad (Solve block) is shown in Fig. 7: reasonable initial approximations to the solution are set, constraints are introduced (these are equations in this case, but, in general, inequalities may be included) and the Mathcad built-in function Find is called, which returns the numerical values of its arguments, which turn the equations into identities. Or, rather, almost in identities since the left and right sides of the equations differ by a small amount. Numerical methods for solving problems have another nameapproximate methods.

The equations are:

- The length of the closed chain L remains constant and consists of two parts: the part lying on the cylinder (So) and the sagging part (Sc).

- The closed line describing the shape of the chain covering the cylinder (see Fig. 2) is continuous (Fo = $\mathrm{Fc}$ ) at the point of separation of the chain from the circle.

- This closed line is smooth $\left(\mathrm{F}^{\prime} \mathrm{o}=\mathrm{F}^{\prime} \mathrm{c}\right)$ at the point of separation of the chain from the circle.

In Fig. 8, it is possible to see the graphical representation of the solution of the chain problem on the cylinder for different ratios of the lengths of the closed chain and of the circumference of the cylinder on which the chain is thrown. The calculations were carried out for $\mathrm{R}=1 \mathrm{~m}$, but the similarity theory can be applied to this problem, and it can be argued that the specific values of $\mathrm{R}$ and $\mathrm{L}$ do not affect the shape of the chain sag - only their ratio is important here. This statement has so far been proved by the authors only by a series of numerical experiments and requires theoretical confirmation.

\section{Finding a Graphic Dependency}

The "chain oval" (as the authors propose to name the family of curves shown in Fig. 1, 2 and 8) also has two diameters - small (horizontal d equal to 2R) and large (vertical $\mathrm{D}$ equal to $\mathrm{R}+\mathrm{h}-\mathrm{a}$ - see Fig. 2). Let's connect these three parameters of the chain circle $(\mathrm{R}, \mathrm{L}$ and $\mathrm{D})$, first graphically, and then analytically. Note that two semi-axes are usually distinguished in an ellipse, and not two diameters (double the value of the semi-axes), but diameters will be used in this paper that will not affect the results.

The Find function in the Solve block of Mathcad is capable of not only returning numerical values (see Fig. 7), but also generating functions. This will help us solve the problem of the chain on the cylinder graphically - see Fig. 9.

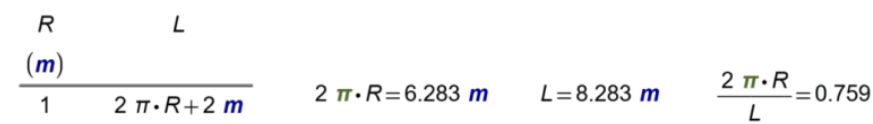

Fig. 6. The Input Data of the Problem of the Chain on a Cylinder.

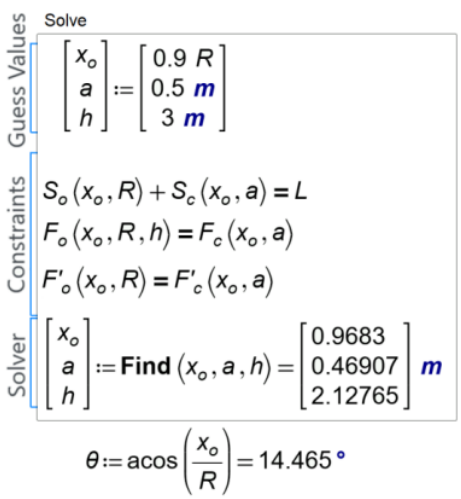

Fig. 7. Solution of the Problem of the Chain on the Cylinder.

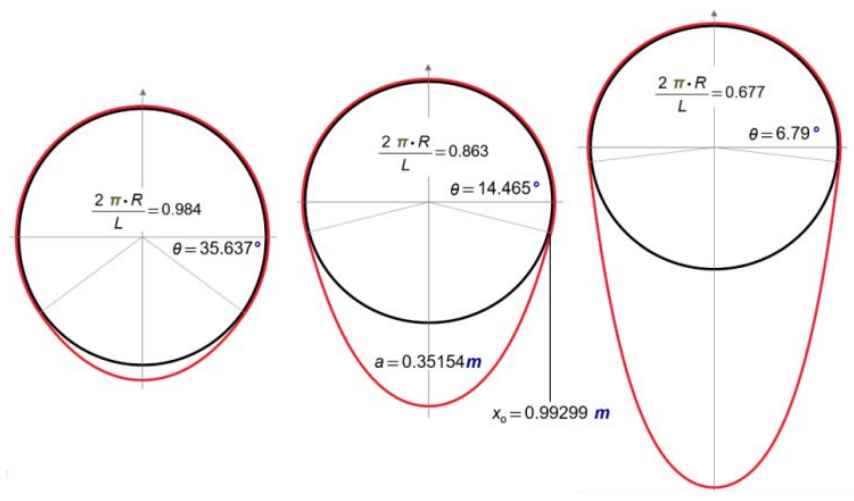

Fig. 8. Graphic Representation of the Solution of the Problem of the Chain on the Cylinder.

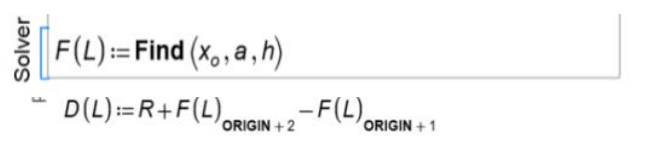$$
L:=2 \pi R, 2 \pi R+0.1 m . .70 \cdot \pi \cdot R
$$

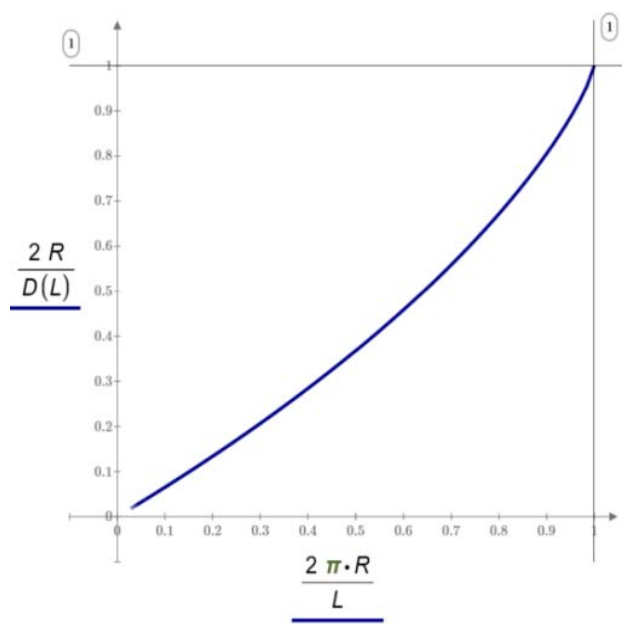

Fig. 9. Graphic Solution to the Problem of a Chain on a Cylinder. 
Fig. 9 shows what changes were made to the Solver area of the Solve block in order to create three functions combined into a vector function with name $\mathrm{F}$, whose first element (the element with the ORIGIN number) is the desired function $\mathrm{xo}(\mathrm{L})$, the second (ORIGIN +1$)$ is the desired function $\mathrm{a}(\mathrm{L})$, and the third (ORIGIN +2 ) is the desired function $h(L)$. The function $\mathrm{D}(\mathrm{L})$, composed of the last two with the addition of the radius of the cylinder, returns the desired value of the large diameter of the chain oval. Having such a function at hand, it is not difficult to construct the corresponding dimensionless graph - see Fig. 9. This curve can be considered a key element in the graphical and analytical solution of the problem of the closed chain thrown around the cylinder.

\section{Finding Approximation Function}

Attempts to analytically solve the chain-to-cylinder problem have been unsuccessful.

Let's solve the chain problem on a cylinder by "Columbus" and find the approximation function.

To do this, let's tabulate the function, whose graph is shown in Fig. 9, using the obtained numerical solution. The obtained data (vectors $\mathrm{X}$ and $\mathrm{Y}$ ) are placed on the website http://zunzun.com, which allows a user to select the leastsquares analytical formula that best approximates the numerical dependence. As a result, the formula $f(x)=$ $a \cdot x /(b+x)+c \cdot x /(d+x)$ and the corresponding numerical values of the coefficients a, b, c and d are obtained. For educational demonstration purposes, the coefficients a, b, c and d could be re-calculated using the Mathcad Prime package.

Fig. 10 shows, firstly, the calculation of the coefficients a, b, c and d itself, for which the built-in genfit function is used general fitting, which requires a first approximation (see the third argument-vector). Secondly, Fig. 10 shows the formation of a function with the name $D$, which returns the value of the large diameter of the chain oval, depending on the value of its small semiaxis $\mathrm{R}$ and the length of the closed chain $\mathrm{L}$. In the same place, it is possible to see the points along which the approximation was carried out, and the smoothing curve itself. The bottom line in Fig. 10 is a calculation of the large diameter of a real chain oval shown in Fig. 1. Its direct measurement (for clarity, graph paper is placed in Fig. 1) gave an answer of 31.4 $\mathrm{cm}$, which is acceptable in accuracy.

The approximating function $\mathrm{D}$ with two arguments $\mathrm{R}$ and L, shown in Fig. 10, can be considered a "pseudo-analytical" solution to the problem of a chain on a cylinder. This solution can also be considered a kind of "Columbian" - a rough solution.
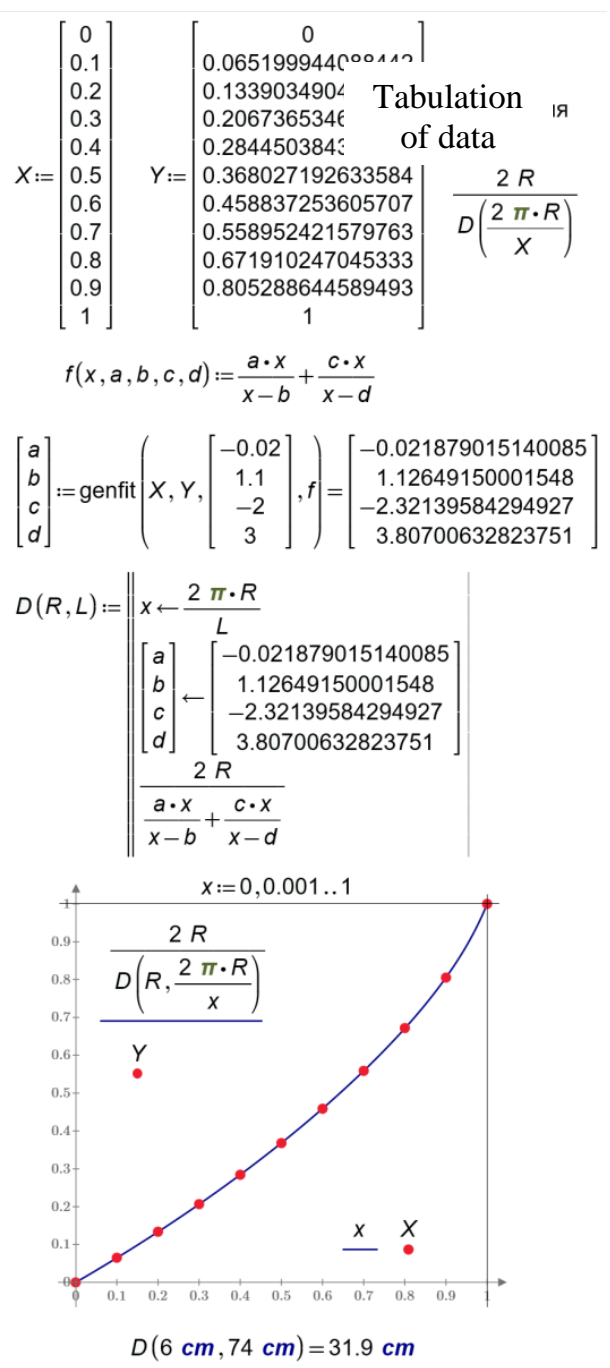

Fig. 10. An Approximating Function for Numerically Solving a Chain Problem on a Cylinder.

\section{Chain With A PENDANT ON A CYLINDER}

Now let's hang a pendant on the chain and see how it will sag on the cylinder [18]. The problem is solved only numerically. In the new problem, there is not one, but two catenaries shifted to the left and right of the ordinate axis at a distance $\Delta x$ - see Fig. 11. In addition, the origin is moved to the center of the circle. This caused the Fc function to have one more additional argument $\mathrm{h}$. Without this change, the minimization mechanism applied to this problem [19-21] will not work. 


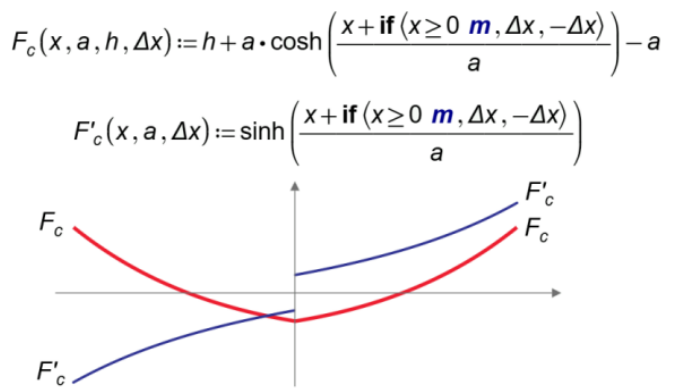

Fig. 11. Catenary Function with a Kink.

To solve this problem, it is needed to add two constants and two functions to the source data:

- a specific (linear) mass of the chain mc;

- a pendant mass m (it is not necessary to enter the value of gravitational acceleration $\mathrm{g}$ - this value is built into Mathcad);

- a function Ycg, which returns the ordinate of the center of gravity of the chain with the pendant (the abscissa of this point is zero, because the problem of the chain with the pendant remains symmetric with respect to the ordinate);

- a PE function that returns the potential energy of a chain with a pendant.

Fig. 12 shows these two additional functions.

The Ycg function has two arguments $\mathrm{x} 1$ and $\mathrm{x} 2$, and not one $\mathrm{x}$ (see Fig. 3). Such a change is aimed at developing the problem, at solving other asymmetric problems, which will be discussed below.

In the problem of a chain with a pendant, a fourth unknown quantity $\Delta \mathrm{x}$ appeared (see Fig. 11), and there were three equations. The solution to the problem is to minimize the potential energy of the chain with the pendant, where these three equations derived in the previous section (see Fig. 7) act as constraints [19-21].

This can be done by replacing the Find function in the Solver area of the Solve block with the Minimize function - see Fig. 13.

Fig. 14 shows a graphical representation of the solution of the chain with a pendant thrown over a cylinder for different values of the pendant mass: $1 \mathrm{~kg}, 20$ grams and 0 (chain without a pendant). In Fig. 14, you can see the dashed chain-a continuation of the real chain without a kink. In the left figure, the catenary is almost a straight line: a heavy load pulls the chain into a string. In the middle figure, the minima of the chain lines are visible at $\mathrm{x}=-0.632 \mathrm{~m}$ and $\mathrm{x}=0.632 \mathrm{~m}$. The right figure is a repetition of Fig. 6. But the two chain lines do not merge into one due to the limited accuracy of the numerical method for solving the problem. The point on the ordinate axis under the circle is the center of gravity of the hanging part of the chain.

Fig. 14 can also be interpreted as follows: assume some ballast is dropped from a balloon, and then the basket is completely detached from it. This is not a chain line, but a catenoid - a surface formed by the rotation of a catenary. Another analogy is that of a soap film that hangs from a ring, and water has accumulated in its lower part.

The problem of the chain without a pendant is simple because it does not take into account the friction force between the chain and the cylinder. However, the assignments suggested below include cases in which this force must be taken into account and others where it is possible to neglect it.

A cylinder with a chain thrown around it with a pendant begins to rotate around its horizontal axis. Determine the angle of rotation of the cylinder at which the chain begins to slip from it. Additionally, a certain coefficient of friction is set.

The cylinder deviates from the horizontal position. Determine the angle of the cylinder at which the chain begins to slide off it.

$$
\begin{gathered}
Y_{c g}\left(x_{1}, x_{2}, a, h, \Delta x\right):=\frac{\int_{x_{1}}^{x_{2}} F_{c}(x, a, h, \Delta x) \cdot \sqrt{1+F_{c}{ }^{\prime}(x, a, \Delta x)^{2}} \mathrm{~d} x}{S_{c}\left(x_{1}, x_{2}, a, \Delta x\right)} \\
P E(x, a, h, \Delta x):=g \cdot S_{c}\left(\begin{array}{c}
-x, x, a, \Delta x) \cdot m_{c} \cdot Y_{c g}(-x, x, a, h, \Delta x)+ \\
+g \cdot m \cdot F_{c}(0 m, a, h, \Delta x)
\end{array}\right.
\end{gathered}
$$

Fig. 12. Functions of the Potential Energy of the Chain with a Pendant.

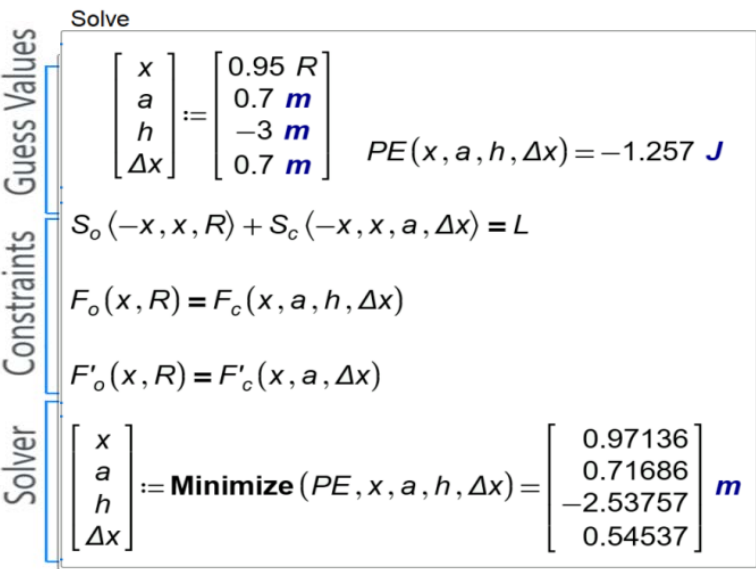

$$
\begin{aligned}
& P E(x, a, h, \Delta x)=-1.063 J \quad \alpha:=\operatorname{acos}\left(\frac{x}{R}\right)=13.746^{\circ}
\end{aligned}
$$

Fig. 13. The Solution to the Problem of the Chain with a Pendant on the Cylinder.
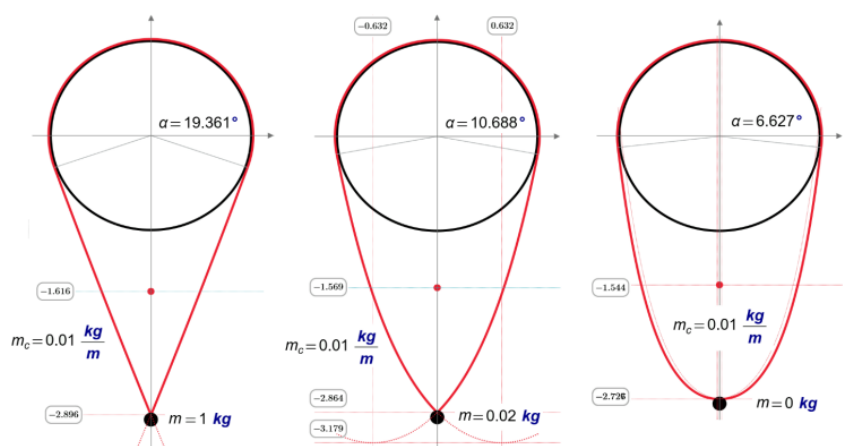

Fig. 14. Graphic Representation of the Solution of the Problem of the Chain with a Pendant on the Cylinder. 
The cylinder deviates from the horizontal position, but the chain is fixed on it. How will the contours of this already asymmetric design change?

These problems could form a basis for the further research.

\section{CONCLUSIONS}

The problem of the parameters of a closed chain (with and without load) thrown over a horizontal cylinder, showing the application of the STEM technology, is considered. Examining together concepts from mathematics, physics and computer science, a numerical solution to the problem is found, a graphical interpretation is obtained, as well as an approximating dependence. The solution of a system of transcendental equations, numerical optimization with constraints and approximation of the numerical solution using the mathematical package Mathcad Prime and online platforms are demonstrated. Additional possible problems for using STEM technology are proposed.

\section{ACKNOWLEDGMENT}

This research was funded by NRU "MPEI" and the Ministry of Science and Higher Education of the Russian Federation (unique identifier RFMEFI60719X0323).

\section{REFERENCES}

[1] E.V. Bodrova, and N.B. Golovanova, "Modernization of the higher technical school: historical experience and prospects," Russian Technological Journal, vol. 5, no. 6, p. 73-97, 2017. DOI: 10.32362/2500-316X-2017-5-6-73-97.

[2] A. Pears, E. Barendsen, V. Dagienè, V. Dolgopolovas, E. Jasutè. "Holistic STEAM Education Through Computational Thinking: A Perspective on Training Future Teachers. In: S. Pozdniakov, V. Dagienè (eds) Informatics in Schools. New Ideas in School Informatics,” ISSEP 2019, Lecture Notes in Computer Science, vol. 11913, Springer, Cham, 2019. DOI:10.1007/978-3-030-33759-9_4.

[3] M. Khine, S. Areepattamannil, "STEAM education: Theory and practice,” Springer, 2019. DOI:10.1007/978-3-030-04003-1.

[4] C. Conradty, S.A. Sotiriou, F.X. Bogner, "How Creativity in STEAM Modules Intervenes with Self-Efficacy and Motivation,” Educ Sci, 2020, 10,70 .

[5] M.-L. How, W.L.D. Hung, "Educing AI-Thinking in Science, Technology, Engineering, Arts, and Mathematics (STEAM) Education," Educ Sci, 2019, 9, 184.

[6] Y. Li, A.H. Schoenfeld, A.A. di Sessa et al., "On Thinking and STEM Education,” Journal for STEM Educ Res, vol. 2, 2019. DOI: 10.1007/s41979-019-00014-x.
[7] S.E. Shadle, A. Marker and B. Earl, "Faculty drivers and barriers: laying the groundwork for undergraduate STEM education reform in academic departments," IJ STEM Ed, vol. 4, no. 8, 2017. DOI: 10.1186/s40594017-0062-7.

[8] B. Yıldırım, and S. Sidekli, "STEM applications in mathematics education: The effect of STEM applications on different dependent variables,” Journal of Baltic Science Education, vol. 17, 2018.

[9] J. Hallström, and K.J. Schönborn, "Models and modelling for authentic STEM education: reinforcing the argument,' IJ STEM Ed, vol. 6, no. 22, 2019. DOI:10.1186/s40594-019-0178-z.

[10] D.J. Shernoff; S. Sinha, D.M. Bressler et al., "Assessing teacher education and professional development needs for the implementation of integrated approaches to STEM education," IJ STEM Ed, vol. 4, no. 13, 2017. DOI: $10.1186 / \mathrm{s} 40594-017-0068-1$.

[11] V. Ochkov, I. Vasileva, M. Nori, K. Orlov, and E. Nikulchev, "Symbolic Computation to Solving an Irrational Equation on Based Symmetric Polynomials Method,” Computation, vol. 8, no. 2, p. 40, 2020. DOI: $10.3390 /$ computation8020040.

[12] V. Ochkov, " $2^{5}$ Problems for STEM Education," Chapman and Hall/CRC, 2020.

[13] L. Bin, L. Yinghui, and Y. Xuegang, "Dynamic modeling and simulation of flexible cable with large sag," Appl Math Mech, vol. 21, p. 707, 2000. DOI: 10.1007/BF02460190.

[14] S. Nedev, "The catenary - an ancient problem on a computer screen," Eur. J. Phys., vol. 21, pp. 451-457, 2000.

[15] B. Maxfield, "Essential PTC Mathcad Prime 3.0. A Guide for New and Current Users,” Academic Press: Cambridge, 2013. DOI: 10.1016/C2012-0-06456-3.

[16] A. Bedford, and W. Fowler, "Engineering Mechanics: Statics,” Addison Wesley, Massachusetts, 1996.

[17] V. Blasjo, "Transcendental Curves in the Leibnizian Calculus. Studies in the History of Mathematical Enquiry,” Academic Press, 2017.

[18] N. Kapustin, and A. Polosin, "On a mixed problem for oscillation of a heavy chain with loads,” AIP Conference Proceedings, 2015. DOI: 10.1063/1.4936721.

[19] R. Almeida, D. Tavares, and D.F.M. Torres. "The Calculus of Variations. In: The Variable-Order Fractional Calculus of Variations. SpringerBriefs in Applied Sciences and Technology,” Springer, Cham, 2019.

[20] Y. Toklu, G. Bekdas, and R. Temur, "Analysis of cable structures through energy minimization. Structural Engineering \& Mechanics 2017, 62, pp. 749-758. DOI:10.12989/sem.2017.62.6.749.

[21] W.-J. Chen, G.-S. Du, and X.-Q. Ren, “Minimization iteration procedure of potential energy and structural analysis for the tension cable-strut structure," Chinese Journal of Computational Mechanics, vol. 27, pp. 1001-1005, 2010. 